\title{
Asymmetric List Sizes in Bipartite Graphs
}

\author{
Noga Alon, Stijn Cambie and Ross J. Kang®
}

\begin{abstract}
Given a bipartite graph with parts $A$ and $B$ having maximum degrees at most $\Delta_{A}$ and $\Delta_{B}$, respectively, consider a list assignment such that every vertex in $A$ or $B$ is given a list of colours of size $k_{A}$ or $k_{B}$, respectively. We prove some general sufficient conditions in terms of $\Delta_{A}$, $\Delta_{B}, k_{A}, k_{B}$ to be guaranteed a proper colouring such that each vertex is coloured using only a colour from its list. These are asymptotically nearly sharp in the very asymmetric cases. We establish one sufficient condition in particular, where $\Delta_{A}=\Delta_{B}=\Delta, k_{A}=\log \Delta$ and $k_{B}=(1+o(1)) \Delta / \log \Delta$ as $\Delta \rightarrow \infty$. This amounts to partial progress towards a conjecture from 1998 of Krivelevich and the first author. We also derive some necessary conditions through an intriguing connection between the complete case and the extremal size of approximate Steiner systems. We show that for complete bipartite graphs these conditions are asymptotically nearly sharp in a large part of the parameter space. This has provoked the following. In the setup above, we conjecture that a proper list colouring is always guaranteed

- if $k_{A} \geq \Delta_{A}^{\varepsilon}$ and $k_{B} \geq \Delta_{B}^{\varepsilon}$ for any $\varepsilon>0$ provided $\Delta_{A}$ and $\Delta_{B}$ are large enough;

- if $k_{A} \geq C \log \Delta_{B}$ and $k_{B} \geq C \log \Delta_{A}$ for some absolute constant $C>1$; or

- if $\Delta_{A}=\Delta_{B}=\Delta$ and $k_{B} \geq C(\Delta / \log \Delta)^{1 / k_{A}} \log \Delta$ for some absolute constant $C>0$.
\end{abstract}

These are asymmetric generalisations of the above-mentioned conjecture of Krivelevich and the first author, and if true are close to best possible. Our general sufficient conditions provide partial progress towards these conjectures.

N. Alon: Supported in part by NSF grant DMS-1855464, BSF grant 2018267 and the Simons Foundation

S. Cambie and R. J. Kang: Supported by a Vidi grant (639.032.614) of The Netherlands Organisation for Scientific Research (NWO). 


\section{Introduction}

List colouring of graphs, whereby arbitrary restrictions on the possible colours used per vertex are imposed, was introduced independently by Erdős, Rubin and Taylor [10] and by Vizing [16]. Let $G=(V, E)$ be a simple, undirected graph. For a positive integer $k$, a mapping $L: V \rightarrow\left(\begin{array}{c}\mathbb{Z}^{+} \\ k\end{array}\right)$ is called a $k$-listassignment of $G$; a colouring $c: V \rightarrow \mathbb{Z}^{+}$is called an $L$-colouring if $c(v) \in L(v)$ for any $v \in V$. We say $G$ is $k$-choosable if for any $k$-list-assignment $L$ of $G$ there is a proper $L$-colouring of $G$. The choosability $\operatorname{ch}(G)$ (or choice number or list chromatic number) of $G$ is the least $k$ such that $G$ is $k$-choosable.

Note that if $G$ is $k$-choosable then it is properly $k$-colourable. On the other hand, Erdős, Rubin and Taylor [10] exhibited bipartite graphs with arbitrarily large choosability. Let $K_{n_{1}, n_{2}}$ denote a complete bipartite graph with part sizes $n_{1}$ and $n_{2}$.

Theorem 1. [10] For some function $M(k)$ with $M(k)=2^{k+o(k)}$ as $k \rightarrow \infty$,

(i) $K_{n, n}$ is not $k$-choosable if $n \geq M(k)$, and

(ii) $K_{n_{1}, n_{2}}$ is $k$-choosable if $n_{1}+n_{2}<M(k)$.

More succinctly Theorem 1 says that $\operatorname{ch}\left(K_{n, n}\right) \sim \log _{2} n$ as $n \rightarrow \infty$.

There are two contrasting ways to try to strengthen this last statement. First does the lower bound hold more generally; that is, does a bipartite graph with minimum degree $\delta$ have choosability $\Omega(\log \delta)$ as $\delta \rightarrow \infty$ ? Indeed this was shown by the first author [1] using a probabilistic argument; later Saxton and Thomason [15] proved the asymptotically optimal lower bound of $(1+$ $o(1)) \log _{2} \delta$. Second does the upper bound hold more generally; that is, does a bipartite graph with maximum degree $\Delta$ always have choosability $O(\log \Delta)$ as $\Delta \rightarrow \infty$ ? Krivelevich and the first author conjectured this in 1998 .

Conjecture 2. [2] There is some absolute constant $C>0$ such that any bipartite graph of maximum degree at most $\Delta$ is $k$-choosable if $k \geq C \log \Delta$.

Johansson's result for triangle-free graphs [12] gives the conclusion with $k \geq$ $C \Delta / \log \Delta$, which is far from the conjectured bound. Conjecture 2 is an elegant problem in a well-studied area, but apart from constant factors ${ }^{1}$ there has been no progress until now.

Despite the apparent difficulty of Conjecture 2, we propose an asymmetric refinement. Given a bipartite graph $G=(V=A \cup B, E)$ with parts $A, B$ and positive integers $k_{A}, k_{B}$, a mapping $L: A \rightarrow\left(\begin{array}{l}\mathbb{Z}^{+} \\ k_{A}\end{array}\right), B \rightarrow\left(\begin{array}{l}\mathbb{Z}^{+} \\ k_{B}\end{array}\right)$ is called a $\left(k_{A}, k_{B}\right)$-list-assignmentof $G$. We say $G$ is $\left(k_{A}, k_{B}\right)$-choosable if there is guaranteed a proper $L$-colouring of $G$ for any such $L$.

Problem 3. Given $\Delta_{A}$ and $\Delta_{B}$, what are optimal choices of $k_{A} \leq \Delta_{A}$ and $k_{B} \leq \Delta_{B}$ for which any bipartite graph $G=(V=A \cup B, E)$ with parts $A$ and $B$ having maximum degrees at most $\Delta_{A}$ and $\Delta_{B}$, respectively, is $\left(k_{A}, k_{B}\right)$ choosable?

\footnotetext{
${ }^{1}$ In a recent advance, Molloy [14] considerably improved on the constant in Johansson's, cf. also [5].
} 
We have the upper bounds on $k_{A}, k_{B}$, since the problem is trivial if $k_{A}>\Delta_{A}$ or $k_{B}>\Delta_{B}$.

Note that Problem 3, since it has a higher-dimensional parameter space, has wider scope than Conjecture 2 and is necessarily more difficult. However, the extra generality in Problem 3 has permitted a glimpse at an unexpected and basic connection between list colouring and combinatorial design theory (Theorem 6). This has prompted us to explore specific areas of the parameter space in Problem 3, motivating concrete versions of Problem 3 in the spirit of Conjecture 2 (Conjecture 7). One hope of ours is that further study of these problems may yield insights into Conjecture 2. But in fact, already in the present work, we have obtained asymmetric progress towards Conjecture 2 (Corollary 10).

Our first main result provides general progress towards Problem 3.

Theorem 4. Let the positive integers $\Delta_{A}, \Delta_{B}, k_{A}, k_{B}$, with $k_{A} \leq \Delta_{A}$ and $k_{B} \leq \Delta_{B}$, satisfy one of the following conditions.

(i) $k_{B} \geq\left(e k_{A} \Delta_{B}\right)^{1 / k_{A}} \Delta_{A}$.

(ii) $e\left(\Delta_{A}\left(\Delta_{B}-1\right)+1\right)\left(1-\left(1-1 / k_{B}\right)^{\Delta_{A} \min \left\{1, k_{B} / k_{A}\right\}}\right)^{k_{A}} \leq 1$.

Then any bipartite graph $G=(V=A \cup B, E)$ with parts $A$ and $B$ having maximum degrees at most $\Delta_{A}$ and $\Delta_{B}$, respectively, is $\left(k_{A}, k_{B}\right)$-choosable.

Theorem 4 under condition $(i)$ follows from a simple application of the Lovász Local Lemma, as we show in Sect. 2. This sufficient condition for Problem 3 is related to independent transversals in hypergraphs, cf. $[8,11]$, and to singleconflict chromatic number [7]. In the most asymmetric settings (when $k_{A}$ and $\Delta_{A}$ are fixed constants), condition $(i)$ is sharp up to a constant factor. We prove Theorem 4 under condition $(i i)$ in Sect. 3 with the Lovász Local Lemma and a link to the coupon collector problem. In an attempt to clear up the 'parameter soup' arising from Problem 3 and the above sufficient conditions, we will discuss specific natural context for these after presenting some further results.

We complement our sufficient conditions for $\left(k_{A}, k_{B}\right)$-choosability with necessary ones, given mainly by the complete bipartite graphs. An easy boundary case is provided as follows, for warmup. This generalises a classic non- $k$ choosable construction.

Proposition 5. For any $\delta, k \geq 2$, the complete bipartite graph $G=(V=A \cup$ $B, E)$ with $|A|=\delta^{k}$ and $|B|=k$ is not $(k, \delta)$-choosable.

Proof. Let the vertices of $B$ be assigned $k$ disjoint lists of length $\delta$, and let the vertices of $A$ be assigned all possible $k$-tuples drawn from these $k$ disjoint lists.

This is best possible in the sense that the conclusion does not hold if $|A|<\delta^{k}$ or $|B|<k$; however, in Sect. 8 we exhibit a non-complete non- $(k, \delta)$-choosable construction that is a slightly more efficient (in the sense that it has $\Delta_{A}=k$ and $\left.\Delta_{B}<\delta^{k}\right)$. On the other hand, Proposition 5 shows that condition $(i)$ in 
Theorem 4 cannot be relaxed much in general. This follows for instance by considering the most asymmetric case, namely $k=k_{A}=\Delta_{A}$ and $\delta=\Delta_{B}^{1 / k}$, with $k$ fixed and $\Delta_{B} \rightarrow \infty$. Thus for fixed $k_{A}$ and $\Delta_{A}$ Problem 3 is settled up to a constant factor.

Our second main result is a related but much broader necessary condition for Problem 3, also via the complete case. Let us write $\bar{M}\left(k_{1}, k_{2}, \ell\right)$ for the hypergraph Turán number defined as the minimum number of edges in a $k_{2^{-}}$ uniform hypergraph on $\ell$ vertices with no independent set of size $\ell-k_{1}$. This parameter is equivalent to the extremal size of approximate Steiner systems; in particular, $\bar{M}\left(k_{1}, k_{2}, \ell\right)$ is equal to the cardinality of a smallest $k_{1}-\left(\ell, \ell-k_{2}, \mathbb{Z}^{+}\right)$ design, cf. [9, Ch. 13] and [13]. (Recall that a $t-\left(v, k, \mathbb{Z}^{+}\right)$design is a collection of $k$-element subsets, called blocks, of some $v$-element set $X$ such that each $t$-element subset of $X$ is contained in at least one block.) We can draw a link between this classical extremal parameter and Problem 3. We show the following result in Sect. 4.

Theorem 6. Let $k_{A}, k_{B}, \ell, k_{1}, k_{2}$ be integers such that $k_{A}, k_{B} \geq 2$ and $\ell=k_{1}+$ $k_{2}+1$. The complete bipartite graph $G=(V=A \cup B, E)$ with $|A|=\bar{M}\left(k_{1}, k_{A}, \ell\right)$ and $|B|=\bar{M}\left(k_{2}, k_{B}, \ell\right)$ is not $\left(k_{A}, k_{B}\right)$-choosable.

This link allows us, using known results for $\bar{M}$, to read off decent necessary conditions for specific parameterisations of Problem 3. As one example, a lower bound on $\operatorname{ch}\left(K_{n, n}\right)$ of the form $\operatorname{ch}\left(K_{n, n}\right) \gtrsim \frac{1}{2} \log _{2} n$ follows from Theorem 6 with the choice $k_{1}=k_{2}=k-1, k_{A}=k_{B}=k$, and $\ell=2 k-1$ for some $k$. As another example, a slightly weaker form of the necessary condition of Proposition 5 follows with the choice $k_{1}=k_{A}\left(k_{B}-1\right), k_{2}=k_{A}-1$, and $\ell=k_{A} k_{B}$. We detail both of these easy examples in Sect. 4 .

Note though that these last two examples show that Theorem 6 provides suboptimal necessary conditions for $\left(k_{A}, k_{B}\right)$-choosability even in the complete case. Furthermore, in Sect. 8 we give a construction to show that the complete case cannot, in general, be precisely extremal for Problem 3. Nevertheless, we surmise that Theorem 6 provides some good rough borders for Problem 3. More specifically, in Sect. 5 we give some basic sufficient conditions for $\left(k_{A}, k_{B}\right)$ choosability specific to the complete case and show in Sect. 6, through some routine asymptotic calculus, how these roughly match with Theorem 6 over a broad family of parameterisations for Problem 3. Then, just as Conjecture 2 was informed by Theorem 1 , the asymptotic behaviour of $\left(k_{A}, k_{B}\right)$-choosability in the complete case leads us to conjecture the following concrete versions of Problem 3.

Conjecture 7. Let the positive integers $\Delta_{A}, \Delta_{B}, k_{A}, k_{B}$ satisfy one of the following.

(i) Given $\varepsilon>0$, we have $\Delta_{A}, \Delta_{B} \geq \Delta_{0}$ for some $\Delta=\Delta_{0}(\varepsilon)$, and

$$
k_{A} \geq \Delta_{A}^{\varepsilon} \quad \text { and } \quad k_{B} \geq \Delta_{B}^{\varepsilon} \text {. }
$$

(ii) For some absolute constant $C>1$,

$$
k_{A} \geq C \log \Delta_{B} \quad \text { and } \quad k_{B} \geq C \log \Delta_{A} .
$$


(iii) $\Delta_{A}=\Delta_{B}=\Delta$, and, for some absolute constant $C>0$,

$$
k_{B} \geq C(\Delta / \log \Delta)^{1 / k_{A}} \log \Delta \quad \text { or } \quad k_{A} \geq C(\Delta / \log \Delta)^{1 / k_{B}} \log \Delta .
$$

Then any bipartite graph $G=(V=A \cup B, E)$ with parts $A$ and $B$ having maximum degrees at most $\Delta_{A}$ and $\Delta_{B}$, respectively, is $\left(k_{A}, k_{B}\right)$-choosable.

We note that in Sect. 5 we also show how this conjecture holds for the complete bipartite graphs (Theorem 15). Conjecture 7 constitutes three natural asymmetric analogues of Conjecture 2. The first is weaker than Conjecture 2; the latter two are stronger. We discuss these separately in turn.

Behind condition $(i)$, Conjecture 7 concerns the question, for what positive functions $f$ does $k_{A} \geq f\left(\Delta_{A}\right)$ and $k_{B} \geq f\left(\Delta_{B}\right)$ suffice for $\left(k_{A}, k_{B}\right)$ choosability, no matter how far apart $\Delta_{A}$ and $\Delta_{B}$ are? Essentially we posit that some $f$ with $f(x)=x^{o(1)}$ as $x \rightarrow \infty$ will work, and this would be best possible due to the complete case (Theorem 16(i)). In particular, $f(x)=O(\log x)$ is impossible, in contrast to Conjecture 2. But in fact, Theorem 4 implies that Conjecture 7 under condition $(i)$ reduces to its most symmetric form.

Corollary 8. Given $0<\varepsilon<1$ and integers $\Delta_{A}, \Delta_{B}$ satisfying $\Delta_{B}>\Delta_{A}^{2 / \varepsilon}$ and $\Delta_{A}>(4 / \varepsilon)^{1 / \varepsilon}$, any bipartite graph $G=(V=A \cup B, E)$ with parts $A$ and $B$ having maximum degrees at most $\Delta_{A}$ and $\Delta_{B}$, respectively, is $\left(\Delta_{A}^{\varepsilon}, \Delta_{B}^{\varepsilon}\right)$ choosable.

As a consequence, Conjecture 7 under condition $(i)$ follows from the same assertion under the further assumption that $\Delta_{A}=\Delta_{B}=\Delta$.

Proof. It suffices to check condition $(i)$ of Theorem 4 with $k_{A}=\Delta_{A}^{\varepsilon}$ and $k_{B}=\Delta_{B}^{\varepsilon}$. Indeed, as $\Delta_{B}^{2}>e \Delta_{A}^{\varepsilon} \Delta_{B}, \varepsilon / 4>1 / \Delta_{A}^{\varepsilon}$ and $\Delta_{B}^{\varepsilon / 2}>\Delta_{A}$, we have $\Delta_{B}^{\varepsilon}>\left(e \Delta_{A}^{\varepsilon} \Delta_{B}\right)^{1 / \Delta_{A}^{\varepsilon}} \Delta_{A}$.

Assume condition $(i)$ of Conjecture 7 , and moreover assume the truth of the conjecture only for its most symmetric form $\Delta_{A}=\Delta_{B}=\Delta$. We may assume that $\Delta_{0}>(4 / \varepsilon)^{1 / \varepsilon}$, and so, without loss of generality, we may also assume by the first part that $\Delta_{A} \leq \Delta_{B} \leq \Delta_{A}^{2 / \varepsilon}$. For any bipartite graph $G=(V=A \cup B, E)$ with parts $A$ and $B$ having maximum degrees at most $\Delta_{A}$ and $\Delta_{B}$, we have from the assumption that $G$ is $k$-choosable for, say, $k=\Delta_{B}^{\varepsilon^{3}} \leq \Delta_{A}^{2 \varepsilon^{2}}$, provided $\Delta_{B}$, and thus $\Delta_{A}$, is large enough as a function of $\varepsilon$. This is smaller than $\Delta_{A}^{\varepsilon}$ for $\Delta_{A}$ sufficiently large as a function of $\varepsilon$, as required.

Thus Conjecture 7 under condition $(i)$ would follow from a weaker form of Conjecture 2. By an earlier work due to Davies, de Joannis de Verclos, Pirot and the third author [5], we so far know that $f(x)=(1+o(1)) x / \log x$ works.

Under condition ( $\mathrm{ii})$, Conjecture 7 concerns a 'crossed' version of the previous question, so for what positive functions $g$ does $k_{A} \geq g\left(\Delta_{B}\right)$ and $k_{B} \geq$ $g\left(\Delta_{A}\right)$ suffice for $\left(k_{A}, k_{B}\right)$-choosability? In this case, we conjecture that $g(x)=$ $O(\log x)$ will work, which coincides with Conjecture 2 in the symmetric case $\Delta_{A}=\Delta_{B}=\Delta$. The complete bipartite graphs demonstrate the hypothetical sharpness of this assertion up to a constant factor for nearly the entire range of 
possibilities for $\Delta_{A}$ and $\Delta_{B}$ (Theorem $16(i i)$ ). Some modest partial progress towards Conjecture 7 under condition (ii) follows from Theorem 4.

Corollary 9. Given $\varepsilon>0$, there exists $\delta$ such that for $\Delta_{B}$ large enough, any bipartite graph $G=(V=A \cup B, E)$ with parts $A$ and $B$ having maximum degrees at most $\Delta_{A}$ and $\Delta_{B}$, respectively, is $\left(\delta \log \Delta_{B},(1+\varepsilon) \Delta_{A}\right)-$ and $\left(\log \Delta_{B},(e+\right.$ $\varepsilon) \Delta_{A}$ )-choosable.

Proof. This follows for $\Delta_{B}$ large enough from condition $(i)$ of Theorem 4 with either $k_{A}=\delta \log \Delta_{B}$ and $k_{B}=(1+\varepsilon) \Delta_{A}$ or with $k_{A}=\log \Delta_{B}$ and $k_{B}=(e+\varepsilon) \Delta_{A}$.

Under condition ( iii), Conjecture 7 concerns the setting most closely related to Conjecture 2. It suggests how Problem 3 might behave for the symmetric case $\Delta_{A}=\Delta_{B}=\Delta$. The complete bipartite graphs demonstrate its hypothetical sharpness up to a constant factor for the entire range of possibilities for $k_{A}$, and thus symmetrically $k_{B}$ (Theorem $16(i i i)$ ). Theorem 4 provides the following partial progress towards Conjecture 7 under condition (iii). In fact, this constitutes significant (asymmetric) progress towards Conjecture 2, and is a first concrete step in this longstanding problem.

Corollary 10. Given $\varepsilon>0$, any bipartite graph $G=(A \cup B, E)$ with parts $A$ and $B$ having maximum degree at most $\Delta$ is $\left((1+\varepsilon) \Delta / \log _{4} \Delta, 2\right)-$ and $((1+\varepsilon) \Delta / \log \Delta, \log \Delta)$-choosable for all $\Delta$ large enough.

Proof. This follows for $\Delta$ large enough from condition (ii) in Theorem 4 with $\Delta_{A}=\Delta_{B}=\Delta$ and either $k_{A}=(1+\varepsilon) \Delta / \log _{4} \Delta$ and $k_{B}=2$ or $k_{A}=$ $(1+\varepsilon) \Delta / \log \Delta$ and $k_{B}=\log \Delta$.

In Sect. 7 we are able to nearly completely characterise $\left(k_{A}, k_{B}\right)$ choosability for complete bipartite graphs if $k_{A} \geq \Delta_{A}-1$. In Sect. 9, we give a connection between minimum degree and $\left(k_{A}, k_{B}\right)$-choosability along the lines of [1].

\section{Probabilistic Preliminaries}

We will use the following standard probabilistic tools, cf. [3].

A Chernoff Bound. For $X \sim \operatorname{Bin}(n, p)$ and $\varepsilon \in[0,1], \mathbb{P}(X<(1-\varepsilon) n p) \leq$ $\exp \left(-\frac{\varepsilon^{2}}{2} n p\right)$.

The Lovász Local Lemma. Consider a set $\mathcal{E}$ of (bad) events such that for each $A \in \mathcal{E}$

(i) $\mathbb{P}(A) \leq p<1$, and

(ii) $A$ is mutually independent of a set of all but at most $d$ of the other events. If $e p(d+1) \leq 1$, then with positive probability none of the events in $\mathcal{E}$ occur.

\section{A Sufficient Condition via Transversals}

In this section, we prove Theorem 4 under condition $(i)$. We prefer to state and prove a slightly stronger form. To do 
so, we need some notational setup. Let $H=(V, E)$ be a hypergraph. The degree of a vertex in $H$ is the number of edges containing it. Given some partition of $V$, a transversal of $H$ is a subset of $V$ that intersects each part in exactly one vertex. A transversal of $H$ is called independent if it contains no edge, cf. [8].

Lemma 11. Fix $k \geq 2$. Let $H$ be a $k$-uniform vertex-partitioned hypergraph, each part being of size $\ell$, such that every part has degree sum at most $\Delta$. If $\ell^{k} \geq e(k(\Delta-1)+1)$, then $H$ has an independent transversal.

Let us first show that Lemma 11 implies Theorem 4 under condition $(i)$.

Proof of Theorem 4 under condition $(i)$. Let the parameters $k_{A}, k_{B}, \Delta_{A}, \Delta_{B}$ satisfy condition $(i)$. Let $L$ be a $\left(k_{A}, k_{B}\right)$-list-assignment of $G$. We would like to show that there is a proper $L$-colouring of $G$. We do so by defining a suitable hypergraph $H=\left(V_{H}, E_{H}\right)$.

Let $(w, c)$ be a vertex of $V_{H}$ if $w \in B$ and $c \in L(w)$.

Let $\left(\left(w_{1}, c_{1}\right), \ldots,\left(w_{k_{A}}, c_{k_{A}}\right)\right)$ be an edge of $E_{H}$ whenever there is some $v \in A$ such that $N(v) \supseteq\left\{w_{1}, \ldots, w_{k_{A}}\right\}$ and $L(v)=\left\{c_{1}, \ldots, c_{k_{A}}\right\}$.

Note that $H$ is a $k_{A}$-uniform vertex-partitioned hypergraph, where the parts are naturally induced by each list in $B$ and so are each of size $k_{B}$. We have defined $H$ and its partition so that any independent transversal corresponds to a special partial $L$-colouring of $G$. In particular, it is an $L$-colouring of the vertices in $B$ for which there is guaranteed to be a colour in $L(v)$ available for every $v \in A$, and so it can be extended to a proper $L$-colouring of all $G$.

Every part in $H$ has degree sum at most $\Delta_{B}\left(\begin{array}{c}\Delta_{A}-1 \\ k_{A}-1\end{array}\right) k_{A} ! \leq \Delta_{B} \Delta_{A}^{k_{A}}$, so the result follows from Lemma 11 with $\ell=k_{B}$ and $\Delta=\Delta_{B} \Delta_{A}^{k_{A}}$.

Proof of Lemma 11. Write $H=(V, E)$ and suppose $\ell^{k} \geq e(k(\Delta-1)+1)$. Consider the random transversal $\mathbf{T}$ formed by choosing one vertex from each part independently and uniformly. For each edge $f \in E$, let $A_{f}$ denote the event that $\mathbf{T} \supseteq f$. Note that $\mathbb{P}\left(A_{f}\right) \leq 1 / \ell^{k}$. Moreover $A_{f}$ is mutually independent of a set of all but at most $k(\Delta-1)$ of the other events $A_{f^{\prime}}$. The transversal $\mathbf{T}$ is independent if none of the events $A_{f}$ occur. Since by assumption $e\left(1 / \ell^{k}\right)(k(\Delta-$ $1)+1) \leq 1$, there is a positive probability that $\mathbf{T}$ is independent by the Lovász Local Lemma.

\section{A Sufficient Condition via Coupon Collection}

Proof of Theorem 4 under condition $(i i)$. Let $k_{A}, k_{B}, \Delta_{A}, \Delta_{B}$ satisfy condition (ii). Let $L$ be a $\left(k_{A}, k_{B}\right)$-list-assignment of $G$. We would like to show that there is a proper $L$-colouring of $G$. To this end, colour each vertex $w \in B$, randomly and independently, by a colour chosen uniformly from its list $L(w)$. Let $T_{v, c}$ be the event that $v \in A$ has a neighbour coloured with colour $c$. Let $T_{v}$ be the event that $T_{v, c}$ happens for all $c \in L(v)$. The proof hinges on the following claim, which is related to the coupon collector problem, cf. e.g. [6]. 
Claim. The events $T_{v, c}$, for fixed $v$ as $c$ ranges over all colours in $L(v)$, are negatively correlated. In particular, $\mathbb{P}\left(T_{v}\right) \leq \prod_{c \in L(v)} \mathbb{P}\left(T_{v, c}\right)$.

Proof. We have to prove, for every $I \subset L(v)$, that $\mathbb{P}\left(\forall c \in I: T_{v, c}\right) \leq \prod_{c \in I}$ $\mathbb{P}\left(T_{v, c}\right)$. If some colour $c \in L(v)$ is not in the list of any neighbour of $v$, both sides equal zero and so the inequality holds. So assume this is not the case. We prove the statement by induction on $|I|$. When $|I| \leq 1$ the statement is trivially true. Let $I \subset L(v)$ be a subset for which the statement is true and let $c^{\prime} \in L(v) \backslash I$. We now prove the statement for $I^{\prime}=I \cup\left\{c^{\prime}\right\}$. We have $\mathbb{P}\left(\forall c \in I: T_{v, c}\right) \leq \mathbb{P}\left(\forall c \in I: T_{v, c} \mid \neg T_{v, c^{\prime}}\right)$ as the probability to use a colour in $I$ is larger if in all neighbouring lists the colour $c^{\prime}$ is removed. This is equivalent to

$$
\begin{aligned}
\mathbb{P}\left(\forall c \in I: T_{v, c}\right) & \geq \mathbb{P}\left(\forall c \in I: T_{v, c} \mid T_{v, c^{\prime}}\right) \\
\Longleftrightarrow \mathbb{P}\left(\forall c \in I^{\prime}: T_{v, c}\right) & \leq \mathbb{P}\left(\forall c \in I: T_{v, c}\right) \mathbb{P}\left(T_{v, c^{\prime}}\right)
\end{aligned}
$$

This last expression is at most $\prod_{c \in I^{\prime}} \mathbb{P}\left(T_{v, c}\right)$ by the induction hypothesis, as desired.

For the $i^{\text {th }}$ colour $c_{i}$ in $L(v)$, let the number of occurrences of $c_{i}$ in the neighbouring lists of $v$ be $x_{i}$. Note that $\mathbb{P}\left(T_{v, c_{i}}\right)=1-\left(1-1 / k_{B}\right)^{x_{i}}$. Using $x_{i} \leq \Delta_{A}$ for every $1 \leq i \leq k_{A}$ and the claim, we have

$$
\mathbb{P}\left(T_{v}\right) \leq\left(1-\left(1-\frac{1}{k_{B}}\right)^{\Delta_{A}}\right)^{k_{A}}
$$

Noting that $\sum_{i=1}^{k_{A}} x_{i} \leq k_{B} \Delta_{A}$ and that the function $\log \left(1-\left(1-1 / k_{B}\right)^{x}\right)$ is concave and increasing, Jensen's Inequality applied with the claim implies that

$$
\mathbb{P}\left(T_{v}\right) \leq\left(1-\left(1-\frac{1}{k_{B}}\right)^{k_{B} \Delta_{A} / k_{A}}\right)^{k_{A}} .
$$

Each event $T_{v}$ is mutually independent of all other events $T_{u}$ apart from those corresponding to vertices $u \in A$ that have a common neighbour with $v$ in $G$. As there are at most $\Delta_{A}\left(\Delta_{B}-1\right)$ such vertices besides $v$, the Lovász Local Lemma guarantees with positive probability that none of the events $T_{v}$ occur, i.e. there is a proper $L$-colouring, as desired.

\section{The Complete Case and Steiner Systems}

In this section, we investigate general necessary conditions for $\left(k_{A}, k_{B}\right)$ choosability via the complete bipartite graphs. Inspired in part by Theorem 1 and related work of Bonamy and the third author [4], this leads naturally to the study of an extremal set theoretic parameter. For positive integers $k_{1}, k_{2}, \ell$, we say that a family $\mathcal{F}$ of $k_{2}$-element subsets of [ $\left.\ell\right]$ has Property $A\left(k_{1}, k_{2}, \ell\right)$ (A is for asymmetric) if there is a $k_{1}$-element subset of [ $\left.\ell\right]$ that intersects every set in $\mathcal{F}$. We then define $\bar{M}\left(k_{1}, k_{2}, \ell\right)$ to be the cardinality of a smallest family of $k_{2}$-element subsets of [ $\left.\ell\right]$ that does not have Property $\mathrm{A}\left(k_{1}, k_{2}, \ell\right)$. Note that 
this definition of $\bar{M}$ coincides with the definition given before the statement of Theorem 6 .

Proof of Theorem 6. We define a $\left(k_{A}, k_{B}\right)$-list-assignment $L$ as follows. Let $\mathcal{F}_{1}$ be a family of $\bar{M}\left(k_{1}, k_{A}, \ell\right) k_{A}$-element subsets of [ $\left.\ell\right]$ without Property $\mathrm{A}\left(k_{1}\right.$, $\left.k_{A}, \ell\right)$. Let $\mathcal{F}_{2}$ be a family of $\bar{M}\left(k_{2}, k_{B}, \ell\right) k_{B}$-element subsets of [ $\ell$ ] without Property $\mathrm{A}\left(k_{2}, k_{B}, \ell\right)$. Assign the sets of $\mathcal{F}_{1}$ as lists to the vertices in $A$ and the sets of $\mathcal{F}_{2}$ as lists to the vertices in $B$. Suppose that $c$ is an $L$-colouring. Then $C_{1}=\{c(a) \mid a \in A\}$ intersects every set in $\mathcal{F}_{1}$ and so $\left|C_{1}\right| \geq k_{1}+1$ by assumption and similarly $C_{2}=\{c(b) \mid b \in B\}$ has cardinality $\left|C_{2}\right| \geq k_{2}+1$. So $\left|C_{1}\right|+\left|C_{2}\right| \geq k_{1}+k_{2}+2>\ell$, implying that $c$ cannot be a proper colouring. rem 6 .

Since $\bar{M}\left(\ell-k_{A}, k_{A}, \ell\right)=\left(\begin{array}{c}\ell \\ k_{A}\end{array}\right)$, we have the following corollary of Theo-

Corollary 12. Let $k_{A}, k_{B}, \ell$ be integers such that $k_{A}, k_{B} \geq 2$ and $\ell \geq k_{A}+$ $k_{B}-1$. The complete bipartite graph $G=(V=A \cup B, E)$ with $|A|=\left(\begin{array}{c}\ell \\ k_{A}\end{array}\right)$ and $|B|=\bar{M}\left(k_{A}-1, k_{B}, \ell\right)$ is not $\left(k_{A}, k_{B}\right)$-choosable.

Although they are trivial, the following observations already show that Theorem 6 and Corollary 12 cannot be improved much in general.

- If $k_{A}=k_{B}=k$ and $\ell=2 k-1$, then $\bar{M}\left(k_{A}-1, k_{B}, \ell\right)=\left(\begin{array}{c}2 k-1 \\ k\end{array}\right)=$ $2^{2 k+o(k)}$ and Corollary 12 implies that $K_{\left(\begin{array}{c}2 k-1 \\ k\end{array}\right),\left(\begin{array}{c}2 k-1 \\ k\end{array}\right)}$ is not $k$-choosable. This implies $\operatorname{ch}\left(K_{n, n}\right) \gtrsim \frac{1}{2} \log _{2} n$, which one can compare to the bounds of Theorem 1.

- If $\ell=k_{A} \cdot k_{B}$, then $\bar{M}\left(k_{A}-1, k_{B}, \ell\right)=k_{A}$ and so Corollary 12 implies that $K_{\left(\begin{array}{c}k_{A} \cdot k_{B} \\ k_{A}\end{array}\right), k_{A}}$ is not $\left(k_{A}, k_{B}\right)$-choosable. This produces a necessary condition on $k_{B}$ for $\left(k_{A}, k_{B}\right)$-choosability only slightly weaker than Proposition 5 .

We present several more instances where Theorem 6 and Corollary 12 are nearly sharp in Sect. 6. For this, we will have use for the following estimates for the parameter $\bar{M}$. A version of this result can be found in [9, Ch. 13], but for completeness we present a standard derivation in the appendix.

Theorem 13. Let $k_{1}, k_{2}$, $\ell$ be integers such that $k_{1}, k_{2} \geq 2$ and $\ell \geq k_{1}+k_{2}$. Then

$$
\frac{\ell !\left(\ell-k_{1}-k_{2}\right) !}{\left(\ell-k_{2}\right) !\left(\ell-k_{1}\right) !} \leq \bar{M}\left(k_{1}, k_{2}, \ell\right)<\frac{\ell !\left(\ell-k_{1}-k_{2}\right) !}{\left(\ell-k_{2}\right) !\left(\ell-k_{1}\right) !} \log \left(\begin{array}{c}
\ell \\
k_{1}
\end{array}\right) \text {. }
$$

\section{Sufficient Conditions in the Complete Case}

In this section, we give general sufficient conditions for $\left(k_{A}, k_{B}\right)$-choosability of complete bipartite graphs $K_{a, b}$. Our strategy for establishing $\left(k_{A}, k_{B}\right)$ choosability in this setting is to take a random bipartition of the set of all colours and try to use one part for colouring $A$ and the other part for colouring $B$. This yields the following lemma. 
Lemma 14. Let the reals $0<\varepsilon, p<1$ and positive integers $a, b, k_{A}, k_{B}$ satisfy either

$$
\begin{aligned}
& a p^{k_{A}}+b(1-p)^{k_{B}}<1 \text { or } \\
& \quad \frac{a p^{k_{A}-1}}{(1-\varepsilon) k_{B}}+b \exp \left(-\varepsilon^{2} k_{B} p / 2\right)<1 .
\end{aligned}
$$

Then the complete bipartite graph $G=(V=A \cup B, E)$ with $|A|=a$ and $|B|=b$ is $\left(k_{A}, k_{B}\right)$-choosable.

Proof. Let $L$ be $\left(k_{A}, k_{B}\right)$-list-assignment of $G$. Let $U=\cup_{v \in V} L(v)$, i.e. $U$ is the union of all colour lists. Define a random partition of $U$ into parts $L_{A}$ and $L_{B}$ as follows. For each colour in $U$, randomly and independently assign it to $L_{B}$ with probability $p$ and otherwise assign it to $L_{A}$.

First assume (1). For a given vertex $v \in A$, the probability that $L(v) \cap$ $L_{A}=\emptyset$ is $p^{k_{A}}$. For a given vertex $v \in B$, the probability that $L(v) \cap L_{B}=\emptyset$ is $(1-p)^{k_{B}}$. So the expected number of vertices $v$ which cannot be coloured with the corresponding list $L_{A}$ or $L_{B}$ is equal to $a p^{k_{A}}+b(1-p)^{k_{B}}$. So the probabilistic method then guarantees some choice of the parts $L_{A}$ and $L_{B}$ such that every vertex in $A$ can be coloured with a colour from $L_{A}$ and every vertex in $B$ with a colour from $L_{B}$.

Otherwise assume (2). For a given vertex $w \in B$, the random variable $\left|L(w) \cap L_{B}\right|$ has a binomial distribution of parameters $k_{B}$ and $p$. So by a Chernoff bound the probability that $\left|L(w) \cap L_{B}\right|$ is smaller than (1 $\varepsilon) k_{B} p$ is smaller than $\exp \left(-\varepsilon^{2} k_{B} p / 2\right)$. Thus, with probability greater than $1-b \exp \left(-\varepsilon^{2} k_{B} p / 2\right)$, we have $\left|L(w) \cap L_{B}\right| \geq(1-\varepsilon) k_{B} p$ for all $w \in B$.

For a given vertex $v \in A$, the probability that $L(v) \cap L_{A}=\emptyset$ is $p^{k_{A}}$. So the expected number of vertices $v$ for which this holds is $p^{k_{A}}|A|$. Thus by Markov's inequality, the probability that there are at least $(1-\varepsilon) k_{B} p$ such vertices $v$ is smaller than $\frac{p^{k} A^{-1} a}{(1-\varepsilon) k_{B}}$.

Due to (2), the probabilistic method guarantees a fixed choice of partition of $U$ into parts $L_{A}$ and $L_{B}$ such that $\left|L(w) \cap L_{B}\right| \geq(1-\varepsilon) k_{B} p$ for every $w \in B$ and the number of vertices $v \in A$ such that $L(v) \cap L_{A}=\emptyset$ is smaller than $(1-\varepsilon) k_{B} p$. Colour any vertex $v \in A$ having $L(v) \cap L_{A}=\emptyset$ with an arbitrary colour from $L(v)$. Colour any other vertex $v^{\prime} \in A$ with a colour from $L\left(v^{\prime}\right) \cap L_{A}$. Finally colour any vertex $w \in B$ with some colour in $L(w) \cap L_{B}$ unused by any vertex of $A$ - this is possible as there were fewer than $(1-\varepsilon) k_{B} p$ colours of $L_{B}$ used to colour vertices in $A$ and the lists in $B$ all have at least $(1-\varepsilon) k_{B} p$ colours from $L_{B}$.

In either case, we are guaranteed a proper $L$-colouring of $G$, as promised.

\section{Asymptotic Sharpness in the Complete Case}

We next use the results of Sects. 4 and 5 to roughly settle the behaviour of complete bipartite graphs with respect to Problem 3 in several regimes. The 
conditions in Theorems 15 and 16 naturally correspond to the conditions in Conjecture 7 .

Theorem 15. Let the positive integers $a, b, k_{A}, k_{B}$ satisfy one of the following.

(i) For any $\varepsilon>0, a, b \geq \Delta_{0}$ for some $\Delta_{0}=\Delta_{0}(\varepsilon), k_{A}>b^{\varepsilon}$ and $k_{B}>a^{\varepsilon}$.

(ii) For any $t>0, k_{A} \geq \frac{1}{t} \log _{2}(2 a)$ and $k_{B}>2^{t} \log (2 b)$.

(iii) We have that $a=b=\Delta$ and either $k_{B}>8(\Delta /(2 \log (2 \Delta)))^{1 / k_{A}} \log (2 \Delta)$ or $k_{A}>8(\Delta /(2 \log (2 \Delta)))^{1 / k_{B}} \log (2 \Delta)$.

Then the complete bipartite graph $G=(V=A \cup B, E)$ with $|A|=a$ and $|B|=b$ is $\left(k_{A}, k_{B}\right)$-choosable.

Proof. Assume condition $(i)$ and assume without loss of generality that $a \geq b$. Fix $\varepsilon>0$ and take $\Delta_{0}>2$ large enough such that $\varepsilon \Delta_{0}^{\varepsilon}>3$ and $x^{\varepsilon / 3}>\log (2 x)$ for every $x \geq \Delta_{0}$. Now $k_{B} \geq a^{\varepsilon}>a^{2 / b^{\varepsilon}} a^{\varepsilon / 3}>(2 a)^{1 / b^{\varepsilon}} \log (2 b)$. By taking $p=$ $(2 a)^{-1 / b^{\varepsilon}}$, we have that (1) is satisfied and the result follows from Lemma 14 .

Assume condition $(i i)$. Take $p=1 / 2^{t}$. Then $a p^{k_{A}} \leq 1 / 2$ and $b(1-$ $p)^{k_{B}} \leq b \exp \left(-p k_{B}\right)<1 / 2$, so that (1) is satisfied and the result follows from Lemma 14.

Assume condition (iii) and by symmetry assume $k_{B}>8(\Delta /$ $(2 \log (2 \Delta)))^{1 / k_{A}} \log (2 \Delta)$. Take $p=8 \log (2 \Delta) / k_{B}$ and $\varepsilon=1 / 2$. Then $p<$ $(2 \log (2 \Delta) / \Delta)^{1 / k_{A}}$ and $b \exp \left(-\varepsilon^{2} k_{B} p / 2\right)<1 / 2$. Also

$$
\frac{a p^{k_{A}}}{(1-\varepsilon) p k_{B}}=\frac{\Delta p^{k_{A}}}{4 \log (2 \Delta)}<\frac{1}{2} \text {. }
$$

Thus (2) is satisfied and the result follows from Lemma 14.

The conditions are also sharp in some sense.

Theorem 16. For each of the following four conditions, there are infinitely many choices of the positive integers $a, b, k_{A}, k_{B}$ satisfying it such that the complete bipartite graph $G=(V=A \cup B, E)$ with $|A|=a$ and $|B|=b$ is not $\left(k_{A}, k_{B}\right)$-choosable.

(i) Given a monotone real function $g$ satisfying $g(1) \geq 1$ and $g(x)=\omega(1)$ as $x \rightarrow \infty$,

$$
k_{A}>b^{1 / g(b)} \quad \text { and } \quad k_{B}=a .
$$

(ii) For any integer $t \geq 4$, either

$$
k_{A} \leq \frac{\log _{2} a}{t} \quad \text { and } \quad k_{B} \leq \frac{2^{t-1}}{e}(\log b-\log \log a) \leq \frac{\log _{2} a}{t}
$$

or

$$
k_{A} \leq 2^{t} \log _{2} a \quad \text { and } \quad k_{B} \leq \frac{\log _{2} b-\log _{2} \log a}{t+\log _{2} t+3},
$$

where $4 \leq b \leq a$.

(iii) For any fixed integer $k>1$ and $\Delta$ sufficiently large, $\Delta_{A}=\Delta_{B}=\Delta$, $k_{A}=k$ and $k_{B}=c(\Delta / \log \Delta)^{1 / k} \log \Delta$ for some constant $c=c(k)$. 
Proof. Consider condition $(i)$. Take $\delta$ large enough so that $g\left(\delta^{a}\right)>a$. Let $k_{A}=\delta$ and $b=\delta^{a}$ and note that $b^{1 / g(b)}=\delta^{a / g(b)}<\delta=k_{A}$, i.e. $k_{A}^{g(b)}>b$. The result then follows from Proposition 5.

Consider condition $(\mathrm{ii})$. For the former case, let

$$
\ell=\frac{2^{t}}{e t} \log _{2} a \quad \text { and } \quad k_{A}=\frac{\log _{2} a}{t} \geq k_{B}=\frac{2^{t-1}}{e}(\log b-\log \log a) .
$$

Then $\left(\begin{array}{c}\ell \\ k_{A}\end{array}\right) \leq\left(e \ell / k_{A}\right)^{k_{A}}=a$. Also

$$
\begin{aligned}
\left(\begin{array}{c}
\ell \\
k_{B}
\end{array}\right) /\left(\begin{array}{c}
\ell-k_{A}+1 \\
k_{B}
\end{array}\right) & \leq\left(\frac{\ell-k_{B}}{\ell-k_{A}-k_{B}}\right)^{k_{B}} \leq\left(\frac{2^{t}-e}{2^{t}-2 e}\right)^{k_{B}} \leq \exp \left(\frac{2 e}{2^{t}} k_{B}\right) \\
& =\frac{b}{\log a} .
\end{aligned}
$$

Here we used the estimation $(x-e) /(x-2 e) \leq \exp (2 e / x)$ for $x \geq 16$.

For the latter case, choose

$\ell=\left(2^{t}+\frac{1}{t+\log _{2} t+3}\right) \log _{2} a, \quad k_{A}=2^{t} \log _{2} a \quad$ and $\quad k_{B}=\frac{\log _{2} b-\log _{2} \log a}{t+\log _{2} t+3}$.

Then $\left(\begin{array}{c}\ell \\ k_{A}\end{array}\right)=\left(\begin{array}{c}\ell \\ \ell-k_{A}\end{array}\right) \leq\left(e \ell /\left(\ell-k_{A}\right)\right)^{\ell-k_{A}} \leq a$ since

$$
e \ell /\left(\ell-k_{A}\right) \leq e\left(2^{t}\left(t+\log _{2} t+3\right)+1\right) \leq 8 t 2^{t}=2^{t+\log _{2} t+3}
$$

Also

$$
\left(\begin{array}{c}
\ell \\
k_{B}
\end{array}\right) /\left(\begin{array}{c}
\ell-k_{A}+1 \\
k_{B}
\end{array}\right) \leq\left(\frac{e \ell}{\ell-k_{A}}\right)^{k_{B}} \leq \frac{b}{\log a} .
$$

In either case, the result follows from Corollary 12 and Theorem 13.

Consider condition (iii). First we make a computation verifying $\bar{M}((\log m) / 2, m / 2, m)<m$ when $m$ is sufficiently large. By Theorem 13, for $m$ sufficiently large, we have that

$$
\begin{aligned}
\bar{M}((\log m) / 2, m / 2, m) & \leq\left(\frac{m}{m / 2-(\log m) / 2}\right)^{(\log m) / 2} \log \left(\begin{array}{c}
m \\
(\log m) / 2
\end{array}\right) \\
& <\sqrt{m}(\log m)^{2}<m .
\end{aligned}
$$

We choose $m=c(\Delta / \log \Delta)^{1 / k} \log \Delta$, where $c=1 /(4 k(k-1))$. By the above computation, this choice satisfies $\bar{M}((\log m) / 2, m / 2, m) \leq \Delta$ for $\Delta$ large enough. Let $b$ be such that $(k-1) b \log \Delta=(\log m) / 2$, and note that $b \sim 2 c$ as $\Delta \rightarrow \infty$.

Let $G=(A \cup B, E)$ be a complete bipartite graph with

$$
|A|=\bar{M}((\log m) / 2, m / 2, m) \quad \text { and } \quad|B|=b \log \Delta\left(\begin{array}{c}
\lceil m /(b \log \Delta)\rceil \\
k
\end{array}\right) .
$$

Note that as $\Delta \rightarrow \infty$

$$
|B| \leq b \log \Delta \frac{\lceil m /(b \log \Delta)\rceil^{k}}{k !} \sim \frac{1}{k !}\left(\frac{c}{b}\right)^{k} b \Delta \leq \Delta
$$

and thus $\Delta_{A}, \Delta_{B} \leq \Delta$ for all $\Delta$ large enough. 
We define a $\left(k_{A}, k_{B}\right)$-list-assignment $L$ as follows. Let $\mathcal{F}$ be a family of $|A|(m / 2)$-element subsets of $[m]$ without Property $\mathrm{A}((\log m) / 2, m / 2, m)$ and assign the sets of $\mathcal{F}$ as lists to the vertices in $A$. So there is no $((\log m) / 2)$ element subset of $[\mathrm{m}]$ that intersects every list in $A$. For $B$, arbitrarily partition $[m]$ into $b \log \Delta$ segments of nearly equal size, and assign as lists to the vertices in $B$ all possible $k$-element subsets chosen from within a single segment.

Note that for any $L$-colouring, $\cup_{w \in B} L(w)$ intersects at least one colour from each $k$-element subset of a segment, and so $\cup_{w \in B} L(w)$ avoids at most $(k-1) b \log \Delta=(\log m) / 2$ colours of $[m]$. However, as noted above, $\cup_{v \in A} L(v)$ must have more than $(\log m) / 2$ colours of $[m]$, and this precludes a proper $L$-colouring.

\section{Sharpness in a Boundary Complete Case}

In this section, we precisely solve Problem 3 for complete bipartite graphs when $k_{A} \geq \Delta_{A}-1$. When $k_{A} \geq \Delta_{A}+1$, we know that any bipartite $G=(A \cup B, E)$ must be $\left(k_{A}, k_{B}\right)$-choosable. The case $k_{A}=\Delta_{A}$ is handled by Proposition 5 and the fact that its conclusion fails if $|A|<\delta^{k}$ or $|B|<k$. The remainder of this section is devoted to the case $k_{A}=\Delta_{A}-1$.

For this it will be useful to have the following simple lemma at our disposal. Given a family $\mathcal{F}$ of disjoint subsets of $X$, a transversal of $\mathcal{F}$ is a subset of $X$ that intersects every set in $\mathcal{F}$ exactly once. An almost-transversal of $\mathcal{F}$ is a subset of $X$ that intersects all but one set in $\mathcal{F}$ exactly once.

Lemma 17. Suppose $\mathcal{F}$ consists of b disjoint sets $F_{1}, F_{2}, \ldots, F_{b}$ such that $\left|F_{1}\right| \geq$ $\left|F_{2}\right| \geq \ldots \geq\left|F_{b}\right|$. Then every almost-transversal of $\mathcal{F}$ is subset of at most $\left|F_{1}\right|$ transversals of $\mathcal{F}$. As a corollary, if $\mathcal{F}_{\star}$ is a family of almost-transversals of $\mathcal{F}$ such that every transversal of $\mathcal{F}$ contains some element of $\mathcal{F}_{\star}$, then $\mathcal{F}_{\star}$ must contain at least $\left|F_{2}\right| \cdots\left|F_{b}\right|$ elements.

Proof. Any almost-transversal of $\mathcal{F}$ can be extended to a transversal of $\mathcal{F}$ by adding exactly one element from $F_{j}$ for some $j \in[b]$, and so there are $\left|F_{j}\right| \leq\left|F_{1}\right|$ choices for this. The corollary follows directly by a union bound argument.

Proposition 18. Let $b \geq 5$ and $\delta=q(b-1)+r$ with $0 \leq r \leq b-2$ and $q$ being integers such that $\delta \gg b$. Then the complete bipartite graph $G=(V=A \cup B, E)$ with $|A|=a$ and $|B|=b$ is $(b-1, \delta)$-choosable if and only if

$$
a<\delta^{b-1}-((b-2) q+r)^{b-1-r}((b-2) q+r-1)^{r} .
$$

Note that for $1 \ll b \ll \delta$, this bound on a is approximately $(1-1 / e) \delta^{b-1}$.

Proof. We write $B=\left\{v_{1}, v_{2}, \ldots, v_{b}\right\}$. Let $L$ be a $(b-1, \delta)$-list-assignment of $G$. If some colour is in the list of at least three different vertices of $B$, or two colours are both in the lists of two disjoint pairs of vertices of $B$, then we can certainly $L$-colour $G$. (The $L$-colouring uses the common colour(s) for the respective vertices and an arbitrary $L$-colouring for the remaining vertices of $B$, followed by a greedy $L$-colouring of the vertices in $A$.) Let $L_{12}=L\left(v_{1}\right) \cap L\left(v_{2}\right)$ 
and analogously define $L_{i j}$ for each $1 \leq i<j \leq b$ and let $\ell_{i j}=\left|L_{i j}\right|$. Consider the family of sets induced by the index pairs of the non-empty $L_{i j}$. This must form an intersecting family, for otherwise we have an $L$-colouring of $G$ similarly as above. We consider two cases depending on this family being trivial or not.

(i) (non-trivial family) Without loss of generality, we may assume that only $L_{12}, L_{13}$ and $L_{23}$ are non-empty among all $L_{i j}$. If there are $b-1$ colours which do not occur as the list of a vertex of $A$, such that the vertices of $B$ can coloured with these $b-1$ colours, then $G$ is $L$-colourable. So if $G$ is not $L$-colourable, every such collection of $b-1$ colours has to occur as the list of a vertex in $A$. There are $\left(\delta-\ell_{12}-\ell_{13}\right)\left(\delta-\ell_{12}-\ell_{23}\right)\left(\delta-\ell_{13}-\right.$ $\left.\ell_{23}\right) \delta^{b-3}$ combinations of $b$ colours which appear only once among the lists $L\left(v_{i}\right), 1 \leq i \leq b$. By Lemma 17 , every $(b-1)$-list $L(a)$ for some $a \in A$ can forbid at most $\delta$ of these and so at least $\left(\delta-\ell_{12}-\ell_{13}\right)\left(\delta-\ell_{12}-\ell_{23}\right)(\delta-$ $\left.\ell_{13}-\ell_{23}\right) \delta^{b-4}$ such vertices are needed to forbid all these colourings. There are also $\left(\ell_{12}+\ell_{13}+\ell_{23}\right) \delta^{b-2}-\left(\ell_{12} \ell_{13}+\ell_{12} \ell_{23}+\ell_{13} \ell_{23}\right) \delta^{b-3}$ other possible colourings of $B$ which do use exactly $b-1$ different colours. So these possible colourings must also be forbidden by some list $L(a)$ if $G$ were not $L$-colourable. This implies that $G$ is $L$-colourable if

$$
\begin{aligned}
a< & \left(\ell_{12}+\ell_{13}+\ell_{23}\right) \delta^{b-2}-\left(\ell_{12} \ell_{13}+\ell_{12} \ell_{23}+\ell_{13} \ell_{23}\right) \delta^{b-3} \\
& +\left(\delta-\ell_{12}-\ell_{13}\right)\left(\delta-\ell_{12}-\ell_{23}\right)\left(\delta-\ell_{13}-\ell_{23}\right) \delta^{b-4} \\
= & \delta^{b-1}-\left(\ell_{12}+\ell_{13}+\ell_{23}\right) \delta^{b-2}+\left(\ell_{12}+\ell_{13}+\ell_{23}\right)^{2} \delta^{b-3} \\
& -\left(\ell_{12}+\ell_{13}\right)\left(\ell_{12}+\ell_{23}\right)\left(\ell_{13}+\ell_{23}\right) \delta^{b-4} .
\end{aligned}
$$

By the AM-GM Inequality, the minimum of the last expression subject to a fixed sum $\ell_{12}+\ell_{13}+\ell_{23}$ occurs when $\ell_{12}=\ell_{13}=\ell_{23}$, and so the minimum is attained when $\ell_{12}=\ell_{23}=\ell_{13}=\delta / 4$, leading to a bound of $\frac{11}{16} \delta^{b-1}$.

(ii) (trivial family) Without loss of generality, we assume only some of the $L_{1 j}$ are non-empty and $\ell_{1 b}=\min \left\{\ell_{1 j} \mid 2 \leq j \leq b\right\}$. There are

$$
\delta^{b-1}-\prod_{j=2}^{b}\left(\delta-\ell_{1 j}\right)
$$

possible ways to choose one colour from every $L\left(v_{i}\right), 2 \leq i \leq b$, in such a way that $L\left(v_{1}\right)$ does contain at least one of these chosen colours. So if $A$ would not contain these combinations among the lists $L(a), a \in A$, one would be able to colour $G$ with these $b-1$ colours. By Lemma 17, one needs also

$$
\left(\delta-\sum_{j=2}^{b} \ell_{1 j}\right) \prod_{j=2}^{b-1}\left(\delta-\ell_{1 j}\right)
$$

lists $L(a), a \in A$, to make it impossible to colour $G$ by first colouring the vertices $B$ solely with colours not appearing among the $L_{1 j}$. Hence the number of disjoint lists $L(a)$ to make a list-colouring of $G$ impossible 
needs to be at least

$$
\delta^{b-1}-\left(\sum_{j=2}^{b-1} \ell_{1 j}\right) \prod_{j=2}^{b-1}\left(\delta-\ell_{1 j}\right) .
$$

This expression is minimised when $\delta-\sum_{j=2}^{b-1} \ell_{1 j}$ and every $\ell_{1 j}$ for $2 \leq j \leq$ $b-1$ are equal to a number of the form $\left\lfloor\frac{\delta}{b-1}\right\rceil$, as by an integral version of the AM-GM Inequality the subtracted product attains its maximum when all factors (which sum to a fixed amount) differ by at most 1 . When $b \ll \delta$ and $b \geq 5$, we have that this is approximately ( $1-(1-$ $\left.1 /(b-1))^{b-1}\right) \delta^{b-1}$, which is smaller than the value $\frac{11}{16} \delta^{b-1}$ obtained in the previous case. Equality can be attained, i.e. $G$ is not $(b-1, \delta)$-choosable when $a=\delta^{b-1}-((b-2) q+r)^{b-1-r}((b-2) q+r-1)^{r}$ (or larger) as we can take the lists as being exactly those mentioned for minimising the expression.

The same analysis also gives the result for $b \in\{3,4\}$. When $b=3$, the bound for $a$ is $\left\lfloor\frac{3}{4} \delta^{2}\right\rceil$. When $b=4$, the same analysis as in Proposition 18 gives that the bound for $a$ occurs in the first case (non-trivial family), resulting in the following detailed proposition.

Proposition 19. Let $\delta \geq 2$. The complete bipartite graph $G=(V=A \cup B, E)$ with $|A|=a$ and $|B|=4$ is $(3, \delta)$-choosable if and only if

$$
a<\left\{\begin{array}{lll}
\frac{11}{16} \delta^{3} & \text { if } \delta \equiv 0 & (\bmod 4), \\
\frac{11}{16} \delta^{3}+\frac{3}{16} \delta+\frac{1}{8} & \text { if } \delta \equiv 1 & (\bmod 4), \\
\frac{11}{16} \delta^{3}+\frac{1}{4} \delta & \text { if } \delta \equiv 2 & (\bmod 4), \\
\frac{11}{16} \delta^{3}+\frac{3}{16} \delta-\frac{1}{8} & \text { if } \delta \equiv 3 & (\bmod 4) .
\end{array}\right.
$$

\section{Sharper than Complete Bipartite}

In this section, we prove that complete bipartite graphs are not exactly extremal for Problem 3. The complete bipartite graph $K_{\delta^{k}-1, k}$ is $(k, \delta)$-choosable, but there are bipartite graphs with $\Delta_{A}=k$ and $\Delta_{B}$ smaller than $\delta^{k}-1$ which are not $(k, \delta)$-choosable.

Proposition 20. For any $\delta, k \geq 2$, there is a bipartite graph $G=(V=A \cup B, E)$ with parts $A$ and $B$ having maximum degrees $k$ and $f(\delta, k)<\delta^{k}$, respectively, that is not $(k, \delta)$-choosable. Moreover, $f(\delta, k) \leq \sum_{i=1}^{\delta} i^{k-1}$.

Proof. We recursively construct bipartite graphs $G_{i}=\left(A_{i} \cup B_{i}, E_{i}\right)$ with parts $A_{i}$ and $B_{i}$ having maximum degree $k$ and $\sum_{j=1}^{i}(\delta-j+1)^{k-1}$. We simultaneously define a $(k, \delta)$-list-assignment $L_{i}$ of $G_{i}$ such that there is some vertex $b_{i} \in B_{i}$ which can only be given one of $\delta-i$ colours out of its list in any proper $L_{i}$-colouring.

Let $G_{1}$ be the complete bipartite graph $K_{\delta^{k-1}, k}$, write $B_{1}=\left\{v_{1}, \ldots, v_{k}\right\}$. For the vertices of $B_{1}$, we assign $k$ disjoint lists of length $\delta$, specifically, 
$L\left(v_{j}\right)=\{(j-1) \delta+1, \ldots, j \delta\}$ for $j \in[k]$. For the vertices of $A_{1}$, we assign as lists all possible $k$-tuples drawn from $\{1\} \times \prod_{j=2}^{k} L\left(v_{j}\right)$. Since $b_{1}:=v_{1}$ cannot be given the colour 1 in any proper $L_{1}$-colouring, the conditions are satisfied for $i=1$.

For the recursion, assume $i \geq 1$ and take the disjoint union of $k$ copies of $G_{i}$, relabelling their $(k, \delta)$-list-assignments so that their colour palettes are mutually disjoint. So the parts $A_{i+1}, B_{i+1}$ of the bipartition so far include the disjoint unions of the $k$ respective parts. Let $v_{1}, \ldots, v_{k}$ be the $k$ copies of $b_{i}$, and for each $j \in[k]$ write $L^{\prime}\left(v_{j}\right)$ for the set of $\delta-i$ colours to which the colour of $v_{j}$ is restricted by assumption. By relabelling, we may assume $1 \in L^{\prime}\left(v_{1}\right)$. We now add $(\delta-i)^{k-1}$ new vertices to $A_{i+1}$ that are adjacent to every $v_{j}$. For these new vertices, we assign as lists all possible $k$-tuples drawn from $\{1\}$ and $L^{\prime}\left(v_{j}\right)$ for $2 \leq j \leq k$. This completes the definition of $G_{i+1}$ and $L_{i+1}$. By induction, $b_{i+1}:=v_{1}$ may only be given a colour from $L^{\prime}\left(v_{1}\right) \backslash\{1\}$ in any proper $L_{i+1}$-colouring, and moreover $b_{i+1}$ is of maximum degree in $B_{i+1}$. So $\left(G_{i+1}, L_{i+1}, b_{i+1}\right)$ satisfies the required conditions. This completes the recursive step.

The graph $G:=G_{\delta}$ with parts $A=A_{\delta}$ and $B=B_{\delta}$ is not $(k, \delta)$ choosable, since by construction we may not give any colour to $b_{\delta}$ in any proper $L_{\delta}$-colouring. Furthermore, the maximum degrees in $A$ and $B$ are respectively $k$ and $\sum_{i=1}^{\delta}(\delta-i+1)^{k-1}=\sum_{i=1}^{\delta} i^{k-1}$, as required.

\section{Degrees and $\left(k_{A}, k_{B}\right)$-Choosability}

In this section, we give a condition on the minimum degree for concluding that a bipartite graph is not $\left(k_{A}, k_{B}\right)$-choosable. This is a reduction to the behaviour for complete bipartite graphs.

Theorem 21. Suppose the complete bipartite graph $G_{0}=\left(V=A_{0} \cup B_{0}, E\right)$ with $\left|A_{0}\right|=a$ and $\left|B_{0}\right|=b$ is not $\left(k_{A}, k_{B}\right)$-choosable. Then any bipartite graph $G=(V=A \cup B, E)$ with parts $A$ and $B$ such that $|A| \leq|B|$ and $B$ has minimum degree $\delta_{B}>4 a b \log 4 a \log k_{A}$ is not $\left(k_{A}, k_{B}\right)$-choosable.

Proof. Let $\mathcal{F}_{a}$ and $\mathcal{F}_{b}$ be the collections of lists of sizes $a$ and $b$, respectively, that can be assigned to $A_{0}$ and $B_{0}$, respectively, to certify non- $\left(k_{A}, k_{B}\right)$ choosability of $G_{0}$. Let $p=1 /\left(4 b \log k_{A}\right)$. Randomly choose $X \subset A$, each vertex included independently with probability $p$. Then $\mathbb{E}(|X|)=p|A|$ and so by Markov's inequality,

$$
\mathbb{P}(|X|>2 p|A|)<\frac{1}{2} .
$$

Define a list-assignment $L_{X}$ of $X$, by assigning to every vertex of $X$ uniformly and independently a list of $\mathcal{F}_{a}$. Call a vertex $v$ in $B$ good if every member of $\mathcal{F}_{a}$ occurs as a list on a neighbour (in $X$ ) of $v$. For any $F \in \mathcal{F}_{a}$, let us say that vis not good due to $F$ if $F$ does not as occur as a list on a neighbour of $v$. Note that

$$
\frac{\mathbb{P}(v \text { is not good due to } F)}{a} \leq\left(1-\frac{p}{a}\right)^{\delta_{B}} \leq \exp \left(-\frac{p}{a} \delta_{B}\right)<\frac{1}{4 a}
$$


implying that

$$
\mathbb{P}(v \text { is not good })<\frac{1}{4} .
$$

So by Markov's inequality,

$$
\mathbb{P}(\mid\{v \mid v \text { is not good }\}|>| B \mid / 2) \leq \frac{\mathbb{E}(\mid\{v \mid v \text { is not good }\} \mid)}{|B| / 2}<\frac{1}{2} .
$$

By the probabilistic method, there is some $X \subset A$ and a list-assignment $L_{X}$ of $X$ such that $|X| \leq 2 p|A|$ and there are at least $|B| / 2$ good vertices. Fix this choice and let $B^{*}$ be the set of good vertices.

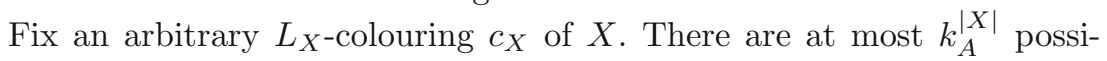
bilities for the colouring $c_{X}$. Define a list-assignment $L_{B^{*}}$ of $B^{*}$, by assigning to every vertex of $B^{*}$ uniformly and independently a list of $\mathcal{F}_{b}$. Since every $v \in B^{*}$ is good, all lists of $\mathcal{F}_{a}$ occur in the neighbourhood of $v$ and at least one choice of a list in $\mathcal{F}_{b}$ would imply that $v$ cannot be properly coloured with a colour of that list. Hence the probability that every $v \in B^{*}$ can be properly

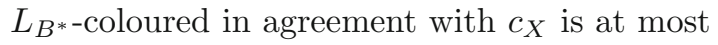

$$
\left(1-\frac{1}{b}\right)^{\left|B^{*}\right|}<\exp \left(-\frac{|B|}{2 b}\right) \text {. }
$$

The probability that some proper colouring of $G$ can be completed given any $L_{X}$-colouring $c_{X}$ is smaller than

$$
k_{A}^{|X|} \exp \left(-\frac{|B|}{2 b}\right) \leq \exp \left(2 p|A| \log k_{A}-\frac{|B|}{2 b}\right) \leq 1 .
$$

Thus by the probabilistic method there exists a list-assignment $L_{B^{*}}$ of $B^{*}$

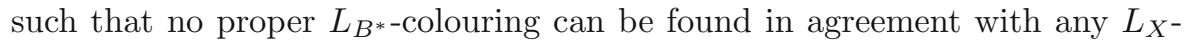
colouring.

\section{Conclusion}

We have begun the investigation of an asymmetric form of list colouring for bipartite graphs. In one direction, we have found good general sufficient conditions through connections to independent transversals and to the coupon collector problem. This has incidentally yielded a non-trivial advance towards a difficult conjecture of Krivelevich and the first author. In another direction, we have established broad necessary conditions through an unexpected link between the bipartite choosability of complete bipartite graphs and a classic extremal set theoretic or design theoretic parameter. This link has fed naturally into the formulation of three attractive conjectures along these lines. Because of the rich connections this problem has to other important areas of combinatorial mathematics, we are hopeful that further study will lead to novel insights. We remark that Conjecture 7 comprises three asymptotic parameterisations of Problem 3 that we found most natural and interesting, all derived essentially from Theorem 6 . There could be several other nice choices. 
Because the terrain is new, there are many interesting angles we have not yet had the opportunity to fully explore.

One possibility, based on the connection to combinatorial design theory, comes to mind. We have that $\bar{M}\left(2, q^{2}, q^{2}+q+1\right)=q^{2}+q+1$ for every prime power $q$ due to the finite projective planes. With a small modification of the substitution of this fact into Corollary 12, we obtain that the complete

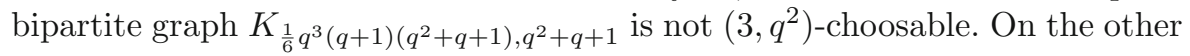
hand, Lemma 14 shows this is not that far from optimal, and in particular (2)

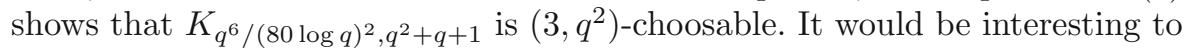
narrow the gap. For the specific case $q=2$, a quick computer search checks that $K_{20,7}$ is not $(3,4)$-choosable, but finding the largest $r$ such that $K_{r, 7}$ is $(3,4)$-choosable seems difficult.

\section{Acknowledgements}

The authors wish to thank the anonymous referees for their careful reading and helpful comments.

Open Access. This article is licensed under a Creative Commons Attribution 4.0 International License, which permits use, sharing, adaptation, distribution and reproduction in any medium or format, as long as you give appropriate credit to the original author(s) and the source, provide a link to the Creative Commons licence, and indicate if changes were made. The images or other third party material in this article are included in the article's Creative Commons licence, unless indicated otherwise in a credit line to the material. If material is not included in the article's Creative Commons licence and your intended use is not permitted by statutory regulation or exceeds the permitted use, you will need to obtain permission directly from the copyright holder. To view a copy of this licence, visit http://creativecommons. org/licenses/by/4.0/.

Publisher's Note Springer Nature remains neutral with regard to jurisdictional claims in published maps and institutional affiliations.

\section{A Extremal Analysis of Approximate Steiner Systems}

Proof of Theorem 13. First we prove the lower bound. Fix a family $\mathcal{F}$ of $k_{2^{-}}$ element subsets of $[\ell]$ with cardinality less than the leftmost expression. Choose $C$ a $k_{1}$-element subset of $[\ell]$ uniformly at random. For any fixed $F \in \mathcal{F}$, we have

$$
\mathbb{P}(F \cap C=\emptyset)=\left(\begin{array}{c}
\ell-k_{2} \\
k_{1}
\end{array}\right) /\left(\begin{array}{c}
\ell \\
k_{1}
\end{array}\right)=\frac{\left(\ell-k_{2}\right) !\left(\ell-k_{1}\right) !}{\ell !\left(\ell-k_{1}-k_{2}\right) !} .
$$

By a union bound and the choice of cardinality of $\mathcal{F}$,

$$
\mathbb{P}(F \cap C=\emptyset \text { for some } F \in \mathcal{F}) \leq \sum_{F \in \mathcal{F}} \mathbb{P}(F \cap C=\emptyset)<1 .
$$

So with positive probability there is a set $C$ certifying that $\mathcal{F}$ has Property $\mathrm{A}\left(k_{1}, k_{2}, \ell\right)$. 
Next we prove the upper bound. Fix $C$ a $k_{1}$-element subset of $[\ell]$. Let $F$ be a $k_{2}$-element subset of $[\ell]$ chosen uniformly at random. Then

$$
\mathbb{P}(F \cap C=\emptyset)=\left(\begin{array}{c}
\ell-k_{1} \\
k_{2}
\end{array}\right) /\left(\begin{array}{c}
\ell \\
k_{2}
\end{array}\right)=\frac{\left(\ell-k_{2}\right) !\left(\ell-k_{1}\right) !}{\ell !\left(\ell-k_{1}-k_{2}\right) !} .
$$

Let $\mathcal{F}=\left\{F_{1}, \ldots, F_{m}\right\}$ be a family of $m k_{2}$-element subsets of $[\ell]$ chosen uniformly at random. Based on the above calculation,

$$
\mathbb{P}\left(F_{i} \cap C \neq \emptyset \text { for all } i \in\{1, \ldots, m\}\right) \leq\left(1-\frac{\left(\ell-k_{2}\right) !\left(\ell-k_{1}\right) !}{\ell !\left(\ell-k_{1}-k_{2}\right) !}\right)^{m} .
$$

There are $\left(\begin{array}{c}\ell \\ k_{1}\end{array}\right)$ choices for $C$, so we have

$\mathbb{P}\left(\mathcal{F}\right.$ contains a $C$ certifying Property $\left.\mathrm{A}\left(k_{1}, k_{2}, \ell\right)\right)$

$$
\leq\left(\begin{array}{c}
\ell \\
k_{1}
\end{array}\right) \exp \left(-m \frac{\left(\ell-k_{2}\right) !\left(\ell-k_{1}\right) !}{\ell !\left(\ell-k_{1}-k_{2}\right) !}\right) \text {. }
$$

This last expression is less than 1 if

$$
m>\frac{\ell !\left(\ell-k_{1}-k_{2}\right) !}{\left(\ell-k_{2}\right) !\left(\ell-k_{1}\right) !} \log \left(\begin{array}{c}
\ell \\
k_{1}
\end{array}\right)
$$

which establishes the upper bound.

\section{References}

[1] N. Alon. Degrees and choice numbers. Random Structures Algorithms, 16(4):364368, 2000.

[2] N. Alon and M. Krivelevich. The choice number of random bipartite graphs. Ann. Comb., 2(4):291-297, 1998.

[3] N. Alon and J. H. Spencer. The probabilistic method. Wiley Series in Discrete Mathematics and Optimization. John Wiley \& Sons, Inc., Hoboken, NJ, fourth edition, 2016.

[4] M. Bonamy and R. J. Kang. List coloring with a bounded palette. J. Graph Theory, 84(1):93-103, 2017.

[5] E. Davies, R. de Joannis de Verclos, R. J. Kang, and F. Pirot. Coloring trianglefree graphs with local list sizes. Random Structures Algorithms, 57(3):730-744, 2020 .

[6] B. Doerr. Probabilistic tools for the analysis of randomized optimization heuristics. In B. Doerr and F. Neumann, editors, Theory of Evolutionary Computation: Recent Developments in Discrete Optimization, pages 1-87. Springer International Publishing, Cham, 2020.

[7] Z. Dvořák, L. Esperet, R. J. Kang, and K. Ozeki. Single-conflict colouring. J. Graph Theory, 97(1):148-160, 2021.

[8] P. Erdős, A. Gyárfás, and T. Łuczak. Independent transversals in sparse partite hypergraphs. Combin. Probab. Comput., 3(3):293-296, 1994. 
[9] P. Erdős and J. Spencer. Probabilistic methods in combinatorics. Academic Press [A subsidiary of Harcourt Brace Jovanovich, Publishers], New York-London, 1974. Probability and Mathematical Statistics, Vol. 17.

[10] P. Erdős, A. L. Rubin, and H. Taylor. Choosability in graphs. In Proceedings of the West Coast Conference on Combinatorics, Graph Theory and Computing (Humboldt State Univ., Arcata, Calif., 1979), Congress. Numer., XXVI, pages 125-157, Winnipeg, Man., 1980. Utilitas Math.

[11] P. E. Haxell. A note on vertex list colouring. Combin. Probab. Comput., 10(4):345-347, 2001.

[12] A. Johansson. Asymptotic choice number for triangle-free graphs. Technical Report 91-5, DIMACS, 1996.

[13] P. Keevash. Hypergraph Turán problems. In Surveys in combinatorics 2011, volume 392 of London Math. Soc. Lecture Note Ser., pages 83-139. Cambridge Univ. Press, Cambridge, 2011.

[14] M. Molloy. The list chromatic number of graphs with small clique number. $J$. Combin. Theory Ser. B, 134:264-284, 2019.

[15] D. Saxton and A. Thomason. Hypergraph containers. Invent. Math., 201(3):925992, 2015.

[16] V. G. Vizing. Coloring the vertices of a graph in prescribed colors. Diskret. Analiz, 29 Metody Diskret. Anal. v Teorii Kodov i Shem:3-10, 101, 1976.

Noga Alon

Department of Mathematics

Princeton University

Princeton NJ08544

USA

e-mail: nogaa@tau.ac.il

and

Schools of Mathematics and Computer Science

Tel Aviv University

6997801 Tel Aviv

Israel

Stijn Cambie and Ross J. Kang

Department of Mathematics

Radboud University

Postbus 9010

6500 GL Nijmegen

The Netherlands

e-mail: ross.kang@gmail.com 
Stijn Cambie

e-mail: stijn.cambie@hotmail.com

Communicated by Bartosz Walczak

Received: 25 April 2020.

Accepted: 17 August 2021. 